CASO CLÍNICO

1. Departamento de Especialidades Quirúrgicas, Instituto Nacional de Salud del Niño San Borja, Lima, Perú

a. Jefe del Servicio de Ginecología Pediátrica y del Adolescente

b. Médico Asistente del Servicio de Cirugía Plástica

c. JefedelDepartamentodeEspecialidades Quirúrgicas

d. Jefe del Servicio de Diagnóstico por Imágenes

e. Asistente del Servicio de Anatomía Patológica

Conflicto de intereses: Los autores manifiestan no tener ningún tipo de conflicto de intereses

Financiamiento: Propio de los autores

Recibido: 19 enero 2021

Aceptado: 7 mayo 2021

Publicación en línea:

Correspondencia:

Mirela Mallqui

- Av.Agustín de la Rosa Toro 1399-San Borja

9iin 973888206/978796802

\. gineco.mimallqui@gmail.com

Citar como: Mallqui M, Wiegering A, Wieslava N, Matos A, Cruzado C. Hiperplasia estromal pseudoangiomatosa (PASH) en niña de 14 años: reporte de caso. Rev Peru Ginecol Obstet. 2021;67(4). DOl: https://doi.org/10.31403/rpgo. v67i2361

\section{Hiperplasia estromal pseudoangiomatosa (PASH) en niña de 14 años: reporte de caso} Pseudoangiomatous stromal hyperplasia (PASH) in a 14-year-old girl: a case report

Mirela Mallqui Mejía1,a, Andrés Wiegering Secchi ${ }^{1, b}$, Normy Wieslava Amiel $^{1, c}$, Aracely Matos Rojas ${ }^{1, d}$, Carla Cruzado Villanueva ${ }^{1, e c}$

DOI: https://doi.org/10.31403/rpgo.v67i2361

\section{RESUMEN}

La hiperplasia estromal pseudoangiomatosa (PASH, por sus siglas en inglés) se presenta entre los 12 y 75 años de edad, siendo prevalente en la edad reproductiva. Se desconoce su etiología. Se caracterizan por ser grandes, deformantes, unilaterales y se les diagnostica por ecografía e histología. En las adolescentes es importante la vigilancia, diagnóstico y manejo tempranos, con la finalidad de evitar cirugías radicales que originen cicatrices grandes, deformidad y asimetría mamaria, que repercuten en el comportamiento y estado emocional de las pacientes. Los tumores de la mama generalmente son benignos en la adolescencia, pero su diagnóstico en este grupo etario es preocupante para la familia y la paciente. Presentamos el caso de una adolescente de 14 años a quien se le realizó abordaje submamario, conservando la simetría mamaria.

Palabras clave. Mama, neoplasias, Adolescente, Ultrasonografía mamaria, Mastectomía segmentaria, Mamoplastia.

\section{ABSTRACT}

Pseudoangiomatous stromal hyperplasia (PASH) occurs between 12 and 75 years of age, being prevalent in the reproductive age. Its etiology is unknown. They are characterized by being large, deforming, unilateral and are diagnosed by ultrasound and histology. In adolescents, early surveillance, diagnosis and management is important in order to avoid radical surgeries that cause large scars, deformity and breast asymmetry, which have repercussions on the behavior and emotional state of the patients. Breast tumors are generally benign in adolescence, but their diagnosis in this age group is worrisome for the family and the patient. We present the case of a 14-year-old adolescent who underwent a submammary approach, preserving breast symmetry.

Key words: Breast neoplasms, Adolescent, Ultrasonography, mammary, Mastectomy, segmental.

\section{INTRODUCCIÓN}

La hiperplasia estromal pseudoangiomatosa es conocida comúnmente como PASH, por sus siglas en inglés. Es una enfermedad benigna rara y actualmente se desconoce su etiología(1). Fue descrita por primera vez en 1986, por Vuitch y col(2). Aparece con frecuencia entre los 12 años y 75 años de edad(3). Se presenta como masa grande nodular y solitaria, firme, indolora, circunscrita, delimitada y móvil, de tamaño variable entre 2 y $13 \mathrm{~cm}$. Frecuentemente unilateral, hay reportes de bilateralidad ${ }^{(4)}$. En algunos casos se le diagnostica en una biopsia. La PASH también puede surgir en la cola axilar de la mama y muy raramente involucra el complejo pezón-areola y el tejido mamario accesorio de la axila(1). El diagnóstico diferencial más importante es con el angiosarcoma(5).

La biopsia excisional es el tratamiento y el diagnóstico final es histológico(6). El diagnóstico, manejo y resultado estético son puntos muy importantes en este grupo etario. Estas tumoraciones grandes son deformantes y requieren vigilancia, diagnóstico y manejo tempranos, con la finalidad de evitar cirugías radicales que originen cicatrices grandes, deformidad y asimetría, que pueden repercutir en el comportamiento y estado emocional de la adolescente. 
Entre los tumores mamarios benignos más frecuentes en la adolescencia se hallan el fibroadenoma juvenil, hipertrofia juvenil (virginal) y la hiperplasia estromal psudoangiomatosa. Los tumores filoides, linfomas, angiosarcomas son extremadamente $\operatorname{raros}^{(7-10)}$

Presentamos el caso de una paciente a quien se le realizó abordaje submamario y conservó la simetría y estética mamaria.

\section{Caso clínico}

Una adolescente de 14 años y 3 meses de edad refirió que notó incremento progresivo en 6 meses del tamaño de su mama derecha, con asimetría de las mamas y sin dolor. Su madre de 42 años era ama de casa y su padre de 43 años, comerciante, ambos aparentemente sanos. La adolescente nació de parto normal, con $3200 \mathrm{~g}$ de peso, tuvo vacunas completas, menarquia a los 11 años, régimen catamenial 4/30, sin dismenorrea.

Al examen de ingreso pesaba $53 \mathrm{~kg}$, talla 1,54 m, índice de masa corporal 22,3, colaboradora durante la evaluación. Se observó regular estado general, de nutrición y de hidratación. La mama derecha, en estadio Tanner 4, estaba ocupada por masa única de bordes definidos de $9 \times 6$ $\mathrm{cm}$, radio 6 hasta radio 12, móvil, no dolorosa, de consistencia dura y que deformaba el pezón y la areola. La mama izquierda, estadio Tanner 4, no mostraba alteraciones. La bioquímica, el examen hematológico y de orina fueron normales. En la ecografía mamaria derecha se observó masa ovalada circunscrita con sombra acústica, de $9 \times 6 \mathrm{~cm}$ en radio 11 y 12 (figura 1).

El tratamiento quirúrgico consistió en la exéresis del tumor (figura 2) mediante abordaje submamario (figura 3). No se evidenció complicaciones durante el postoperatorio, y fue dada de alta luego de 1 día de hospitalizada, con analgésicos, antibióticos y vendaje de mamas. Acudió a control después de 72 horas del postoperatorio para retiro del vendaje. Después de una semana se le retiró las suturas. En el examen físico, la herida operatoria estaba afrontada y sin flogosis.

El informe histológico fue: Tumor pardo grisáceo de $11 \times 7,7 \times 5,5 \mathrm{~cm}$. Diagnóstico final: Hiperplasia estromal pseudoangiomatosa (PASH) (figura
Figura 1 (A, B). EN LA ECOGRAFÍA MAMARIA ANTES DE LA CIRUGÍA, LOS HALLAZGOS SON VARIABLES, PERO EN LA MAYORÍA SE OBSERVA UNA MASA SÓLIDA, CIRCUNSCRITA, DE FORMA OVALADA O REDONDEADA, COMO EN EL CASO PRESENTADO.

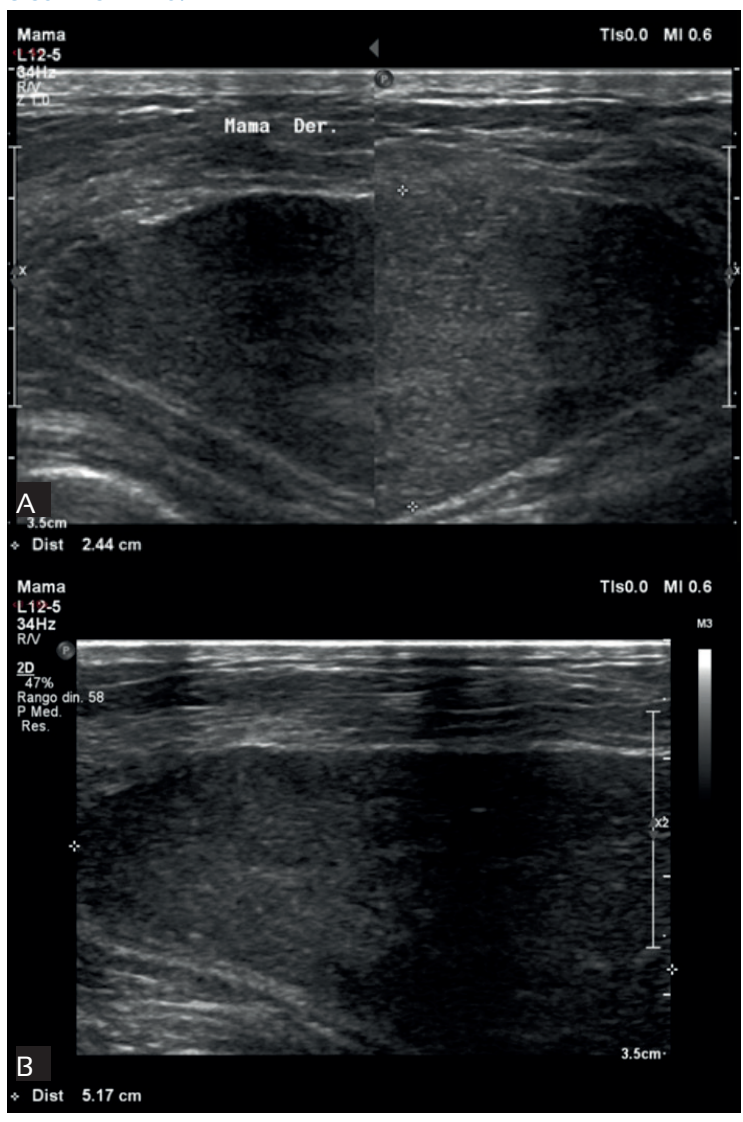

Figura 2. Pieza operatoria: SE APRECiA unA MASA SÓlida de BORDES DEFINIDOS, PARDO GRISÁCEA, DE $11 \times 7,7 \times 5,5 \mathrm{cM}$.

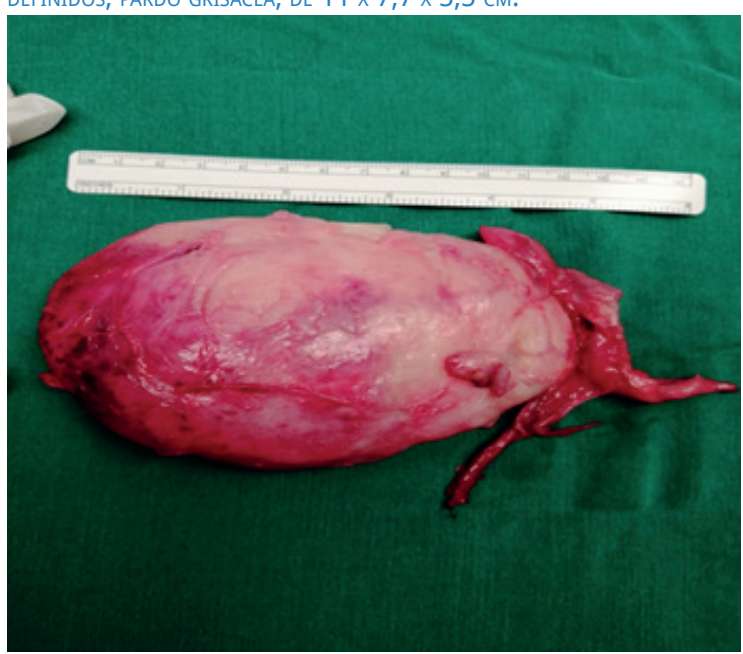

4). Actualmente, la paciente continúa en control y con seguimiento favorable. En el último control de 2021, no evidenció crecimiento de otra tumoración. 
Figura 3. A. Incisión submamaria derecha. B. Control a la semana.

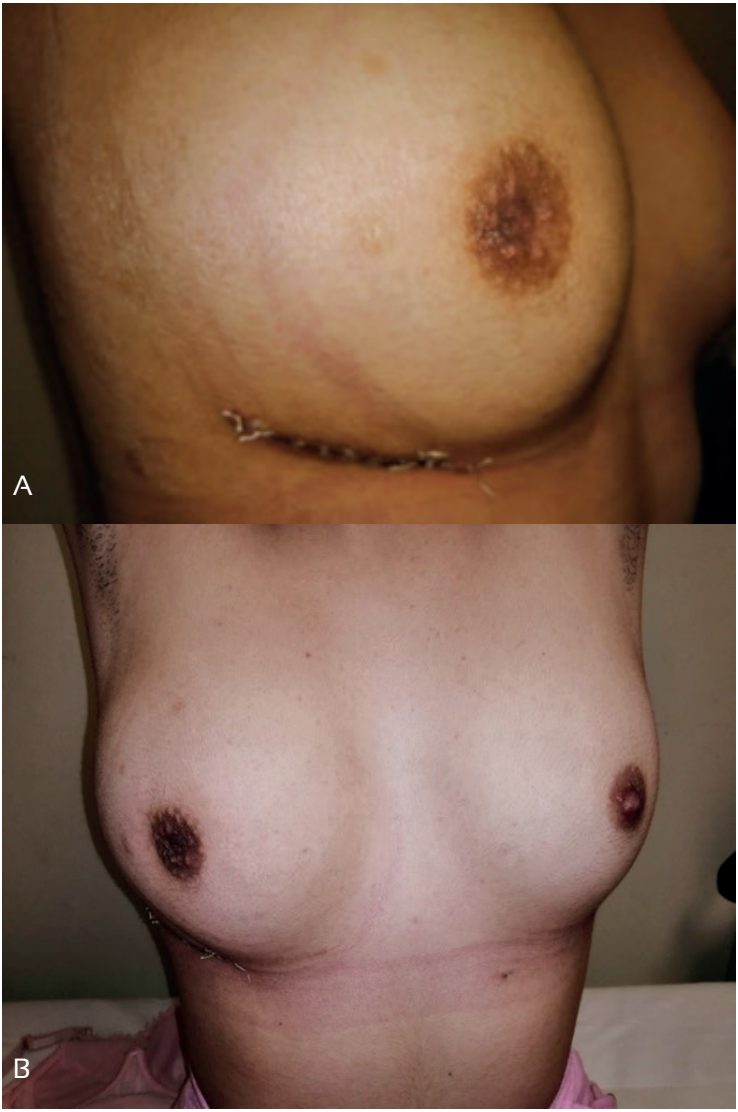

\section{Discusıón}

Se comunica el primer caso de PASH en nuestra institución, siendo importante dar a conocer esta patología, frecuente entre los 12 y 75 años y más prevalente en las mujeres en edad reproductiva. En la adolescencia, generalmente son benignos. Se desconoce la etiología del PASH.

En todo control ginecológico de una adolescente se debe realizar una historia clínica completa, examen físico general y examen de mama. En estos casos, la paciente acudirá a consulta con una tumoración bien delimitada de consistencia dura, no dolorosa, deformante y de rápido crecimiento.

El diagnóstico diferencial de los tumores de mama es fundamental para el manejo de fibroadenomas, fibroadenomas gigantojuveniles, tumor filodes, linfoma. Entre los exámenes de ayuda, la ecografía ${ }^{(11-13,16)}$ de mama en este tipo de tumor es inespecífica, encontrando generalmente una masa sólida, circunscrita, de forma ovalada o redonda. El diagnóstico es anatomopatológico; se describe un parénquima mamario
Figura 4. Descripción histológica: A. Estudio histológico QUe CONFIRMA HIPERPLASIA ESTROMAL PSEUDOANGIOMATOSA: LOS CORTES HISTOLÓGICOS MUESTRAN PARÉNQUIMA MAMARIO CON NUMEROSAS HENDIDURAS ESTROMALES. ESTAS HENDIDURAS ESTÁN REVESTIDAS POR MIOFIBROBLASTOS. LOS ESPACIOS PRODUCIDOS POR LAS HENDIDURAS ESTÁN VACÍOS, SIMULANDO COMPLEJOS VASOS SANGUÍNEOS ANASTOMOSADOS. B. LAS HENDIDURAS RODEAN LOS LÓBULOS DE APARIENCIA NORMAL E INVOLUCRAN EL ESTROMA INTRALOBULILLAR Y EL ESTROMA INTERLOBULILLAR.

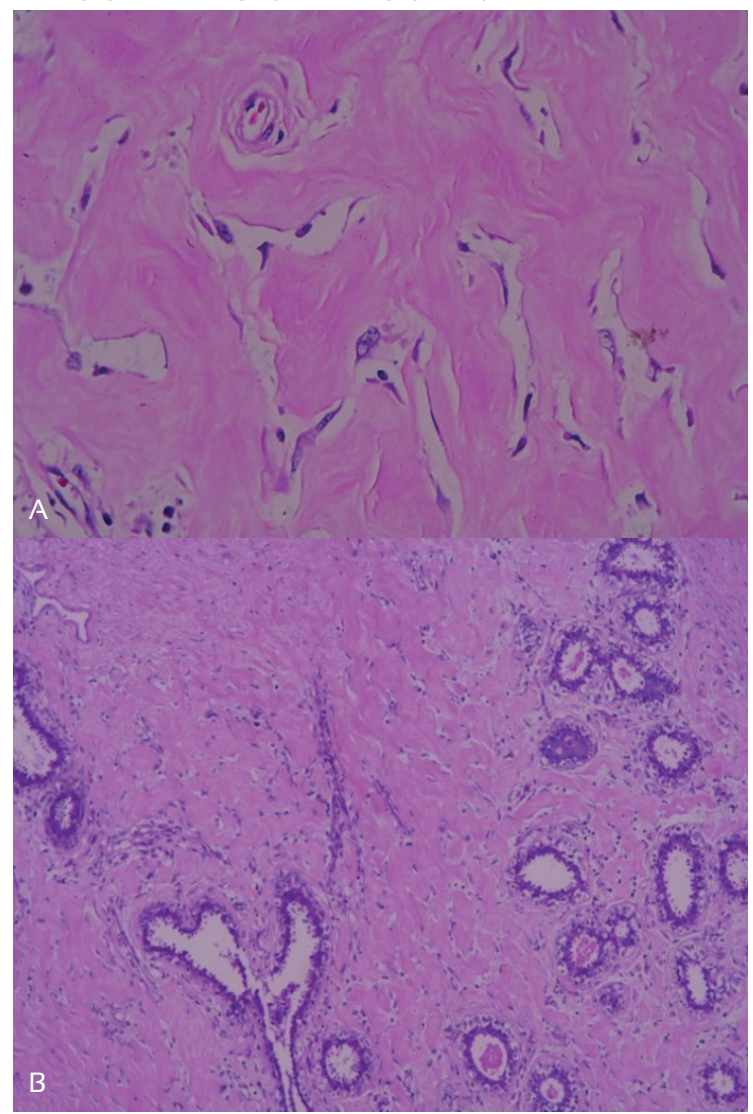

con numerosas hendiduras estromales. Estas hendiduras están revestidas por miofibroblastos ${ }^{(15)}$. Los espacios producidos por las hendiduras están vacíos, simulando complejos vasos sanguíneos anastomosados. Las hendiduras están presentes tanto en el estroma interlobulillar como en el estroma intralobulillar.

En este grupo etario, el abordaje quirúrgico debe ser conservador y adecuado. Se debe realizar seguimiento de las pacientes, porque se presenta recurrencia entre 15 y $22 \%(8,14,16)$.

En conclusión, la hiperplasia estromal pseudoangiomatosa es una lesión que se aprecia generalmente en mujeres en edad reproductiva o como hallazgo incidental como resultado de una biopsia. Pero, también ha sido descrito en niñas y adolescentes como una masa palpable, unilateral firme, no dolorosa, móvil. El diagnóstico es 
mediante imágenes e histología. Es fundamental el diagnóstico diferencial con los fibroadenomas gigantojuveniles y linfomas. El tratamiento quirúrgico debe ser conservador en este grupo etario.

\section{REFERENCIAS BIBLIOGRÁFICAS}

1. Singh KA, Lewis MM, Runge RL, Carlson GW. Pseudoangiomatous stromal hyperplasia. A case for bilateral mastectomy in a 12-year-old girl. Breast J. 2007 Nov-Dec;13(6):603-6. doi: 10.1111/j.1524-4741.2007.00499.x

2. Vuitch MF, Rosen PP, Erlandson RA. Pseudoangiomatous hyperplasia of mammary stroma. Hum Pathol. 1986 Feb;17(2):185-91. doi: 10.1016/s0046-8177(86)80292-1

3. Ferreira M, Albarracin CT, Resetkova E. Pseudoangiomatous stromal hyperplasia tumor: a clinical, radiologic and pathologic study of 26 cases. Mod Pathol. 2008 Feb;21(2):201-7. doi: $10.1038 /$ modpathol.3801003

4. Tumeh RA, Sabino Neto M, Cunha AL, Silva MM de A, de Paula HR, Ferreira LM. Hiperplasia estromal pseudoangiomatosa: caso raro em menina de 11 anos. Rev bras cir plast. 2017;32(2):295-8.

5. Virk RK, Khan A. Pseudoangiomatous stromal hyperplasia: an overview. Arch Pathol Lab Med. 2010 Jul;134(7):1070-4. doi: 10.1043/2008-0686-RS.1

6. Pohlodek K, Minarovjech V, Gál V, Mečiarová I. Tumorous form of pseudoangiomatous stromal hyperplasia of the breast in a young female and a young male. Am J Surg. 2018;35(3):105-9. doi:10.1177/0748806818764152

7. Khokher S, Shahzad R, Shahbaz S. Pseudoangiomatous hyperplasia of breast: Report of two cases presenting with giant tumors and literature review. Qual Prim Care. 2016;24(3):111-3.

8. Jaunoo SS, Thrush S, Dunn P. Pseudoangiomatous stromal hyperplasia (PASH): a brief review. Int J Surg. 2011;9(1):20-2. doi: 10.1016/j.jijsu.2010.09.005

9. Baker M, Chen H, Latchaw L, Memoli V, Ornvold K. Pseudoangiomatous stromal hyperplasia of the breast in a 10-yearold girl. J Pediatr Surg. 2011 Aug;46(8):e27-31. doi: 10.1016/j. jpedsurg.2011.04.063

10. Weinstein SP, Conant EF, Orel SG, Zuckerman JA, Bellah R. Spectrum of US findings in pediatric and adolescent patients with palpable breast masses. Radiographics. 2000 Nov-Dec;20(6):1613-21. doi: 10.1148/radiographics.20.6.g00nv091613

11. Chung EM, Cube R, Hall GJ, González C, Stocker JT, Glassman LM. From the archives of the AFIP: breast masses in children and adolescents: radiologic-pathologic correlation. Radiographics. 2009 May-Jun;29(3):907-31. doi: 10.1148/ rg.293095010

12. Kaneda HJ, Mack J, Kasales CJ, Schetter S. Pediatric and adolescent breast masses: a review of pathophysiology, imaging, diagnosis, and treatment. AJR Am J Roentgenol. 2013 Feb;200(2):W204-12. doi: 10.2214/AJR.12.9560

13. Goel NB, Knight TE, Pandey S, Riddick-Young M, de Paredes ES, Trivedi A. Fibrous lesions of the breast: imaging-pathologic correlation. Radiographics. 2005 NovDec;25(6):1547-59. doi: 10.1148/rg.256045183

14. Irshad A, Ackerman SJ, Pope TL, Moses CK, Rumboldt T, Panzegrau B. Rare breast lesions: correlation of imaging and histologic features with WHO classification. Radiographics. 2008 Sep-Oct;28(5):1399-414. doi: 10.1148/rg.285075743

15. Hoda SA, Rosen PP. Observations on the pathologic diagnosis of selected unusual lesions in needle core biopsies of breast. Breast J. 2004 Nov-Dec;10(6):522-7. https://doi. org/10.1111/j.1075-122X.2004.21412.x

16. Jones KN, Glazebrook KN, Reynolds C. Pseudoangiomatous stromal hyperplasia: imaging findings with pathologic and clinical correlation. Am J Roentgenol. 2010 Oct;195(4):103642. doi: 10.2214/AJR.09.3284 